\title{
Cyniclomyces guttulatus is an opportunistic pathogen in rabbits with coccidiosis
}

Tuanyuan Shi ${ }^{1}$, Hongchao Sun ${ }^{1}$, Yuan Fu ${ }^{1}$, Hao Lili ${ }^{3}$, Yongxue Zhou ${ }^{1}$, Liu Yan ${ }^{1}$, Guolian Bao ${ }^{1 *}$, Xun Suo ${ }^{2 *}$

${ }^{1}$ Department of Animal Parasitology, Institute of Animal Husbandry and Veterinary Medicine, Zhejiang Academy of Agricultural Science, Hangzhou, 310021, China

${ }^{2}$ National Animal Protozoa Laboratory, College of Veterinary Medicine, China Agricultural University, Beijing 100093, China

${ }^{3}$ College of Life Science \& Technology, Southwest Minzu University, Chengdu, 610041, China

${ }^{*}$ Corresponding author.

Guolian Bao

Department of Animal Parasitology, Institute of Animal Husbandry and Veterinary Medicine, Zhejiang Academy of Agricultural Science

Tel./fax: +86571 86400836

E-mail address: baoguolian@163.com

Xun Suo

National Animal Protozoa Laboratory, College of Veterinary Medicine, China Agricultural University, Beijing 100093, China

Tel./fax: +861062734325.

E-mail address: suoxun@,cau.edu.cn

\begin{abstract}
:
Cyniclomyces guttulatus is a common inhabitant of the gastrointestinal tract in rabbits, and large numbers are often present in feces of diarrheic rabbits. However, its relation with rabbit diarrhea has not been clearly identified. We isolated a C. guttulatus Zhejiang strain from a rabbit with severe diarrhea and optimized the culture conditions in YPG medium. The sequenced $18 \mathrm{~S}$ and 26S ribosomal DNA fragments were $1559 \mathrm{bp}$ and $632 \mathrm{bp}$, respectively, and showed $99.8 \%$ homology with the $18 \mathrm{~S}$ ribosomal sequence of the NRRL Y-17561 isolate from the dog and 100\% homology with the 26S ribosomal sequence of the DPA-CGR1 and CGDPA-GP1 isolates from the rabbit and guinea pig. Our isolate was not pathogenic to healthy SPF rabbits. Instead, rabbits inoculated with the yeast had a slightly better body weight gain and
\end{abstract}


higher food intake. Rabbits co-inoculated with $C$. guttulatus and the coccidian, $E$. intestinalis developed more severe coccidiosis as shown by clinical signs, and decreased body weight gain, diarrhea and death, associated with significantly higher fecal output of $C$. guttulatus vegetative cells but lower coccidian oocysts output than the rabbits inoculated with $C$. guttulatus or E. intestinalis alone. We also surveyed the prevalence of C. guttulatus in rabbits and found a positive rate of $83 \%$ in Zhejiang province. Our results indicate that $C$. guttulatus alone is not pathogenic to healthy rabbits, but could become an opportunistic pathogen when the digestive tract is damaged by other pathogens such as coccidia.

Author summary: Cyniclomyces guttulatus, a commensal yeast in rabbit gastrointestinal tract, is very commonly seen in diarrhea cases. However, it is unclear whether it causes or is a co-cause of diarrhea with other pathogens. Here, a $C$. guttulatus Zhejiang strain was firstly isolated from a rabbit with severe diarrhea and the culture conditions in YPG medium were optimized. Then, it was identified in morphology and molecular. It was agreed with the previous description in morphology and showed a closer phylogenetic relationship with other strains originated from herbivores than those from the carnivore. Finally, the C. guttulatus Zhejiang strain was inoculated to SPF rabbits singly or co-inoculated with Eimeria intestinalis. All of the results in animal assays show C. guttulatus alone is not pathogenic but seems a probiotic microorganism in rabbits. However, it could become an opportunistic pathogen when the digestive tract is damaged by other pathogens such as coccidia.

Key words: Rabbit, Cyniclomyces guttulatus, Eimeria intestinalis, opportunistic pathogen, diarrhea

\section{Background}

Diarrhea is very common in rabbits, especially in weanling rabbits, and causes huge losses to rabbitry. According to some reports, more than $50 \%$ of mortalities in rabbits could be caused by intestinal disease with diarrhea ${ }^{[1-3]}$. Currently, more than twenty microorganisms including viruses (e.g. Lapine rotavirus), bacteria (e.g. Esherichia coli, Salmonella typhimurium, Clostridium welchii and Pasteurella multocida) and parasites (e.g. Eimeria spp., Passalurus ambiguus, Cryptosporidium spp. and Giardia duodenalis) have been identified as pathogens causing diarrhea in rabbits ${ }^{[4-15]}$. In addition to the above pathogens, Cyniclomyces guttulatus, a commensal yeast in rabbit gastrointestinal tract is also commonly seen in diarrhea cases. However, it is unclear whether it causes or is a co-cause of diarrhea with other pathogens. Some researchers believed that $C$. guttulatus was not a pathogen causing diarrhea, but is probably a salubrious normal inhabitant based on its common existence in healthy animals and the absence of clinical signs in experimental rabbits inoculated with $C$. guttulatus isolates $^{[16,17]}$. However, other researchers believed it could be an opportunistic pathogen based on the large number of yeast cells in feces of diarrheic 
animals and the positive response of some diarrheic cases to anti-fungal treatment with nystatin ${ }^{[18-22]}$. To clearly establish the relationship between $C$. guttulatus and diarrhea in rabbits, a $C$. guttulatus strain was isolated and identified from a rabbit with severe diarrhea. Then, its relationship to rabbit diarrhea was investigated through inoculation of $C$. guttulatus alone and co-inoculation with an intestinal protozoan, Eimeria intestinalis. In addition, the prevalence of $C$. guttulatus in rabbits was surveyed in Zhejiang province of China.

\section{Materials and methods}

\section{Isolation and cultivation of $C$. guttulatus}

A C. guttulatus Zhejiang strain was isolated from a severely diarrheic rabbit. Approximately 0.5 grams of intestinal content were diluted to about 500 vegetative cells of $C$. guttulatus per milliliter with sterilized distilled water. Then, 20 microliters of the suspension were added to 10 milliliter YPG (pH 1.5) medium supplemented with $100 \mathrm{mg} / \mathrm{L}$ ampicillin. It was cultured on a 96-well culture plate with 100 microlitres of medium per well at $37^{\circ} \mathrm{C}$ with $10 \% \mathrm{CO}_{2}$ for 2 hours. Wells with a single C. guttulatus vegetative cell were further cultured for 5 days before monoclonal cells of C. guttulatus were smeared and cultivated on solid YPG plate ( $\mathrm{pH} 4.5)$ supplemented with $100 \mathrm{mg} / \mathrm{L}$ ampicilin at $37^{\circ} \mathrm{C}$ with $10 \% \mathrm{CO}_{2}$. A single colony of $C$. guttulatus was transferred to liquid YPG medium ( $\mathrm{pH} 4.5)$ and cultivated at $37^{\circ} \mathrm{C}$ on a orbital shaker (Zhichu, China) at a constant rotating speed of $200 \mathrm{rpm}$. Yeast multiplication was monitored using an automatic microbial growth curve analyser (Bioscreen, Finland) and an optical density scanner (Bug Lab, USA).Culture conditions were optimized by varying the medium $\mathrm{pH}$ and culture temperature.

\section{C. guttulatus identification}

The morphology of $C$. guttulatus Zhejiang strain was observed under a light microscope with $400 \times$ magnification. Molecular identification was undertaken by PCR and gene sequencing. Two specific primer pairs for the small subunit (18S) and large subunit (26S) ribosomal RNA genes were synthesized according to Kurtzman CP (1998) ${ }^{[23]}$. The primer pairs were: 18S upper primer (TACGGTGAAACTGCGAATGG), 18S lower primer (GCTGATGACTTGCGCTTACT), 26S upper primer (GCATATCAATAAGCGGAGGAAAAG) and 26S lower primer (GGTCCGTGTTTCAAGACGG). The PCR conditions for the 18S DNA fragment were initial denaturation at $95{ }^{\circ} \mathrm{C}$ for $5 \mathrm{~min}$, followed by 30 cycles of $94{ }^{\circ} \mathrm{C}$ denaturation for $45 \mathrm{sec}$, primer annealing at $55^{\circ} \mathrm{C}$ for $45 \mathrm{sec}$, and extension at $72{ }^{\circ} \mathrm{C}$ for $90 \mathrm{sec}$. A final primer extension of $10 \mathrm{~min}$ at $72{ }^{\circ} \mathrm{C}$ completed the amplification process. The amplification for $26 \mathrm{~S}$ was the same as for $18 \mathrm{~S}$ except for extension at 72 ${ }^{\circ} \mathrm{C}$ for $120 \mathrm{sec}$ during the PCR cycles. C. guttulatus vegetative cells were directly used as the template for PCR. The PCR products were examined and separated by $1 \%$ agarose gel electrophoresis. The target bands were purified by a gel extraction kit and sequenced by Sangon Biotech (Shanghai, China). The sequenced 18S and 26S gene 
fragments of the C. guttulatus Zhejiang isolate were submitted to Genbank with the Bankit procedure and blasted in the NCBI (National Center for Biotechnology Information, USA) database. Similar reference sequences were retrieved. The phylogenetic analysis was performed using the MegAlign program (DNAstar Inc., USA). The phylogenetic tree was constructed using the Clustal W method. Informations about $C$. guttulatus isolates and other yeast species used the construction of the phyogenetic trees are listed in Table 1.

\section{Animals}

Specific-pathogen-free (SPF) rabbits were purchased from Pizhou Dongfang Rabbit Breeding Co., Ltd (Pizhou, China) and reared in our institute. Pre-weaning SPF rabbits were supplied with carrots and $0.5 \%$ milk powder in drinking water. No coccidia oocysts and $C$. guttulatus were detected in feces of these rabbits before use.

\section{Inoculation of Cyniclomyces guttulatus in rabbits and examination of yeast colonization in the gastrointestinal tract}

Three groups of 20 day-old SPF rabbits $(n=4)$ were orally inoculated with $1 \times 10^{6}, 1$ $\times 10^{7}$ or $1 \times 10^{8}$ C. guttulatus vegetative cells per rabbit, and designated as G1, G2 and G3, respectively. Another group (G4) was not inoculated with the yeast and served as the control. Body weight, activities, appetite and excreta were recorded before and after yeast inoculation. Feces from all groups were collected daily and examined for yeast cells under a light microscope.

All rabbits were euthanized 18 days after inoculation. Contents and the mucous layer of the stomach, duodenum, jejunum, and ileum were collected, smeared and microscopically examined. Because the yeast was observed in the stomach (see Results), tissues from different regions of the stomach were immediately frozen for microscopic examination, and fixed in $10 \%$ formalin for preparation of paraffin sections for microscopic observation after staining with PAS (Periodic acid-schiff) and in 5\% glutaraldehyde for preparation of electron-microscope sections for observation by transmission electron microscopy.

\section{Co-infection of Cyniclomyces guttulatus and Eimeria intestinalis in rabbits}

Two groups (designated as CG and CG/EI) of 28 day-old SPF rabbits were orally inoculated with $4 \times 10^{7} \mathrm{C}$. guttulatus cells per rabbit $(\mathrm{n}=4)$. After 14 days, CG/EI and another group (designated as EI, without $C$. guttulatus inoculation) were infected with $1 \times 10^{4}$ sporulated oocysts of $E$. intestinalis. One group (designated as NON) served as un-infected control. Body weight, activities, appetite and excreta were recorded before and after inoculation. Feces were collected for coccidial oocysts and yeast cell counting. E. intestinalis oocysts in feces were counted between 10-16 days after infection using the McMaster method as described previously (Jeffers, 1975). Vegetative cells of $C$. guttulatus were counted 2 days before to 14 days after the infection of E. intestinalis. Briefly, one gram of feces was mashed with a glass stick 
and mixed with 60 milliliter of tap water. The fecal suspension was filtered through a 100-mesh sieve. C. guttulatus cells in the filtrate were counted in a haemocytometer under a light microscope with $100 \times$ magnification.

\section{Ethics statement}

All experimental procedures were approved by the Zhejiang Academy of Agricultural Science Animal Ethics Committee (approval number, 20191904) and due attention was paid to the welfare of the animals. The rabbits were reared under stress-free environment, eliminating strong light and noise, with one rabbit per cage. Physical condition was monitored every day during all experimental procedures. Euthanasia was performed with an intra-cardiac pentobarbital overdose in accordance with the experiment design ${ }^{[24]}$.

\section{Prevalence survey}

Fecal samples were collected from 253 healthy rabbits in four regions including Fuyang, Haining, Deqing and Wencheng (abbreviated names: FY, HN, ZX and WC) in Zhejiang province of China. The sample numbers from FY, HN, DQ and WC were respectively 50, 5049 and 104. Among the surveyed rabbits, 66 were below 60 days old and 187 above 60 days old. The collected samples were stored at $4{ }^{\circ} \mathrm{C}$ and examined within one week. The examination was performed as follows: 2 grams of feces were mixed with 60 milliliter of tap water. The mixture was filtered through a 100-meshsieve. Then, 100 microliter of filtrate was collected for wet mount examination under a light microscope with $100 \times$ magnification. Twenty fields were observed for each sample.

\section{Statistical analysis}

Statistical analyses were performed for $C$. guttulatus cell counts, rabbit body weight, and E. intestinalis oocyst counts by GraphPad Prism 5.01. Data were expressed as mean \pm standard deviation, and the t-test was used to analyse differences between the mean values. Differences between groups with $p$ values $<0.05$ were considered statistically significant.

\section{Results}

\section{A Cyniclomyces guttulatus Zhejiang strain was isolated and culture conditions optimized}

A C. guttulatus Zhejiang strain was isolated from a diarrheic rabbit and cultivated in the YPG medium at low $\mathrm{pH}$ and identified by light microscopy. Microscopically, the vegetative cells of $C$. guttulatus were ellipsoid, colorless and about 20-50 $\mu \mathrm{m}$ in length and were occupied with two large vacuoles in the cytoplasm [Fig. 1 A\&C]. $C$. 
guttulatus formed pseudohyphae when it was cultivated in a stationary culture station [Fig. 1 B]. When it was cultivated with rotation, free vegetative cells were clearly visible. It could aerobically grow at a temperature range of 36 to $42{ }^{\circ} \mathrm{C}$ and a pH range of 1.5 to 4.5 in liquid YPG medium [Fig.1 D\&E]. The optimal culture medium $\mathrm{pH}$ was 4.5 [Fig.1 D]. The logarithmic growth phase was 24 to 60 hours at various temperatures and the $\mathrm{pH}$ value of 4.5 [Fig. $1 \mathrm{E}$ ]. At the culture temperature of $40^{\circ} \mathrm{C}$ and $\mathrm{pH} 4.5$, the cell density of $C$. guttulatus reached $4.62 \times 10^{7} \pm 3.98 \times 10^{6}$ cells per milliliter over $60 \mathrm{~h}$ from the initial culture density of $1 \times 10^{4}$ cells per milliliter [Fig. 1 E].

\section{The $C$. guttulatus Zhejiang strain showed a close relationship with reference strains originated from herbivores}

The sequenced length for the $18 \mathrm{~S}$ fragment of the $C$. guttulatus Zhejiang strain was 1559 bp. It showed $98 \%$ sequence identity and $100 \%$ coverage with that of the $C$. guttulatus NRRL Y-17561 strain reported in Genbank (accession number JQ698886.1)[Fig 2 A]. In the phylogenetic tree based on the 18S fragment, the Zhejiang strain clustered and formed a sister clade with the NRRL Y-17561 strain. The sequenced length for the 26S fragment of the C. guttulatus Zhejiang strain was $632 \mathrm{bp}$, and showed 100\% (95\% coverage), 100\% (93\% coverage), $97.2 \%$ (84\% coverage), $96.6 \%$ (96\% coverage) and $95.9 \%$ (95\% coverage) identity with those of CGDPA-GP1, DPA-CGR1, Dog-1, DPA-CGD1 and NRRL Y-17561 strains, respectively. In the $26 \mathrm{~S}$ phylogenetic tree, the Zhejiang strain was positioned in the same clade with CGDPA-GP1 and DPA-CGR1, and formed a sister clade with Dog-1, DPA-CGD1 and NRRL Y-17561 strains [Fig2 B]. According to data in the Genbank, C. guttulatus CGDPA-GP1 and DPA-CGR1 strains originated from herbivores, guinea pigs and rabbits, respectively, while C. guttulatus Dog-1 and DPA-CGD1 strains originated from the dog. Thus, the C. guttulatus Zhejiang strain showed a closer phylogenetic relationship with the strains originated from herbivores than those from the carnivore.

\section{C. guttulatus Zhejiang strain is non-pathogenic to healthy rabbits}

To study the pathogenicity of $C$. guttulatus, we inoculated SPF rabbits with a large dose of yeast cells. Two days after inoculation, vegetative cells of $C$. guttulatus were detectable in rabbit feces. None of the rabbits inoculated with $1 \times 10^{6}$ to $10^{8}$ of $C$. guttulatus showed clinical signs of illness. Interestingly, C. guttulatus-innoculated groups (G1-G3) had less feed waste than the control group (G4) [Fig3 B\&C]. Mean body weight of inoculated groups was slightly higher than that of the control group although the difference was not statistically significant $(p>0.05)$ [Fig3. A]. Autopsy showed no macroscopic or microscopic lesions in the gastrointestinal tract of the inoculated rabbits despite a large number of $C$. guttulatus cells in the gastric and intestinal contents. Especially, a thick layer of $C$. guttulatus cells colonized the gastric mucosa [Fig3 D-F]. PAS-stained gastric tissue sections showed a dense layer of 
saccharides on the gastric mucosa and also on the cell wall of C. guttulatus [Fig3 G]. The $C$. guttulatus cells probably attached to the stomach mucosa through these filamentous saccharides as shown by transmission electron microscopy [Fig3 H\&I]. Our findings indicate that the C. guttulatus Zhejiang strain colonizes on the gastric mucosa, but it is non-pathogenic to healthy rabbits.

\section{C. guttulatus is an opportunistic pathogen in rabbits infected with Eimeria intestinalis}

We investigated whether $C$. guttulatus could be an opportunistic pathogen in rabbits infected with coccidia. Rabbits pre-inoculated with C. guttulatus (CG/EI group) developed more severe illness and intestinal lesions following the infection of $E$. intestinalis than those non-inoculated with C. guttulatus (EI group). Compared with the EI group, more severe diarrhea, loss of appetite and constipation were observed in the CG/EI group. The number of $C$. guttulatus vegetative cells in feces of the CG/EI group was significantly higher than that of the CG group $(p<0.05)$ on 9 and 10 days post E. intestinalis infection [Fig4 A]; the mean cells per gram of feces in the CG/EI group were $1.22 \times 107 \pm 1.38 \times 106$ on day 9 and $8.55 \times 106 \pm 5.52 \times 10^{5}$ on day 10 , 4.7 fold and 3.2 fold higher than those of the EI group, respectively. In contrast, coccidian reproduction was lower in the co-inoculation rabbits than the rabbits inoculated with $E$. intestinalis alone, as shown by a markedly lower oocyst output in the CG/EI group than in the EI group; the total fecal oocyst count per rabbit on day 10 was $2.87 \times 10^{9} \pm 8.13 \times 10^{7}$ in the co-inoculation group, compared with $4.57 \times 10^{9} \pm 6.83 \times 10^{7}$ in the group without yeast inoculation [Fig4 B]. In addition, the peak oocyst excretion of the CG/EI group was also lower than that of the EI group $\left(1.04 \times 10^{9} \pm 2.45 \times 10^{7}\right.$ on day 11 and $1.61 \times 10^{9} \pm 3.43 \times 10^{7}$ on day 13$)$. Fecal oocyst excretion in the CG/EI group both peaked and cleared earlier than that in the EI group [Fig4 B]. Thus, C. guttulatus proliferation was enhanced by E. intestinalis infection, while $E$. intestinalis reproduction was suppressed by $C$. guttulatus. In addition, one rabbit of the CG/EI group died on the 10th day post infection. The dead rabbit had disseminated hemorrhage and nodules in the lower jejunum and ileum and a large number of E. intestinalis oocysts and $C$. guttulatus vegetative cells were detected in the small intestine [Fig4 D\&E]. In addition, mean body weight of the CG/EI group was slightly lower than the CG group 11 to 17 days post infection [Fig4 C]. In summary, rabbits inoculated with both E. intestinalis and C. guttulatus developed more severe clinical signs and intestinal lesions (and one death) than the rabbits inoculated with E. intestinalis only, associated with greater $C$. guttulatus and lower $E$. intestinalis output. The findings suggest $C$. guttulatus may be an opportunistic pathogen to rabbits with coccidia.

\section{C. guttulatus was highly prevalent in rabbits}

We conducted a survey of rabbits carrying C. guttulatus by analyzing C. guttulatus cells in feces. C. guttulatus was detected in 210 of 253 fecal samples from rabbits above 30 days old in Zhejiang province, a positive rate of $83 \%$. Of the surveyed 
rabbits, the positive rate for rabbits above 60 days old was $69.7 \%(46 / 66)$ and for rabbits less than 60 days old $87.7 \%$ (164/187). The number of rabbits with a high $C$. guttulatus load (>100 cells per microscopic field at 200x magnification) were 59 (23.3\%), consisting of 4 rabbits less than 60 days old and 55 rabbits above 60 days old ( $6.1 \%$ and $29.4 \%$ of the age group, respectively). Thus, C. guttulatus was highly prevalent in healthy rabbits, especially in older rabbits.

\section{Discussion}

Cyniclomyces guttulatus is a monotypic yeast genus of the Saccharomycetaceae family, and inhabits the gastrointestinal tract of many animal species including rabbits, dogs and guinea pigs ${ }^{[17,22,25]}$. C. guttulatus was first described more than 60 years ago, and is commonly found in rabbit feces. A large number of $C$. guttulatus cells are often found in feces of diarrheic rabbits, but it is unknown whether $C$. guttulatus causes diarrhea in rabbits. We isolated a $C$. guttulatus Zhejiang strain from a rabbit with severe diarrhea. At optimized culture $\mathrm{pH}$ and temperature, a single clone was expanded and studied for its pathogenicity in rabbits. We demonstrated that the $C$. guttulatus Zhejiang strain is not a primary pathogen causing diarrhea in healthy SPF rabbits. Inoculation of as high as $1 \times 10^{8}$ vegetative cells per rabbit did not result in any clinical signs of illness or gastrointestinal lesions. This is consistent with previously reported studies, where rabbits orally or intravenously inoculated with $C$. guttulatus isolates showed no clinical signs of illness $^{[16,17,26]}$. Unexpectedly, we found that rabbits inoculated with C. guttulatus showed better performance in terms of body weight gain and food intake. C. guttulatus seems a probiotic microorganism in rabbits, especially in weanling rabbits. However, the findings of massive vegetative cells of $C$. guttulatus commonly seen in feces of rabbits with diarrhea suggest it could be a causative microorganism of GI tract disturbance.

Some authors proposed $C$. guttulatus could be an opportunistic pathogen or play a co-causative role in diarrhea of its host, based on indirect evidence that the antifungal agent, nystatin was effective in the treatment of some diarrheic cases ${ }^{[19-22]}$. In our study, C. guttulatus was proved an opportunist through co-infection with the coccidian species $E$. intestinalis, a parasite causing diarrhea and intestinal lesions in rabbits. Mortality and more severe clinical signs and intestinal lesions were observed in rabbits co-infected with $C$. guttulatus and E. intestinalis than in rabbits inoculated with E. intestinalis only. Compared with rabbits inoculated with C. guttulatus alone, vegetative cells of $C$. guttulatus more prolificly multiplied in the co-infection group, peaking at 9-10 days post $E$. intestinalis infection. This time period is consistent with that of intestinal lesions of $E$. intestinalis infection ${ }^{[27,28]}$. The massive multiplication of $C$. guttulatus was probably a consequence of altered gastrointestinal environment in the rabbits from $E$. intestinalis infection. Compared with rabbits with $E$. intestinalis infection alone, E. intestinalis oocyst excretion in the co-infected rabbits decreased by $37 \%$. We speculate that the rapid and massive multiplication of C. guttulatus vegetative cells could contribute to the severe symptoms in the co-infected rabbits, and the massive multiplication of $C$. guttulatus also limits the reproduction of $E$. 
intestinalis.

In addition, our epidemiological survey showed $C$. guttulatus is prevalent in rabbits in Zhejiang, China. The positive rate in rabbits was as high as $83 \%$. This was significantly higher than that in other host animals such as dogs in which a prevalence of $14-21 \%$ was reported ${ }^{[20,21,29]}$. Coccidia are also highly prevalent in rabbits. According to a previous study, the overall prevalence of rabbit coccidia is $41.9 \%$ in China, and as high as $70 \%$ in some regions ${ }^{[12]}$. So, C. guttulatus may contribute to the morbidity and mortality of rabbits with coccidiosis.

In summary, $C$. guttulatus as a commensal yeast is very common in rabbits. It is usually not pathogenic in healthy rabbits and seems a probiotic microorganism in rabbits, but it could become an opportunistic pathogen when the gastrointestinal environment is altered by enteric pathogens such as coccidia. Considering the high prevalence of both $C$. guttulatus and coccidia in rabbits, the potential harm of $C$. guttulatus to the rabbit industry warrants attention and further study.

\section{Acknowledgements}

This research work was supported by the National Natural Science Foundation of China (Project number 31101810), Project of Zhejiang Province Public Welfare Technology Application Research Project (Project number 2017C32037), China Agricultural Research System (Project number nycytx-44-3-2) and Key Research and Development Plan of Zhejiang Province (Project numbers 2016C02054-10, 2019C02052).

\section{References}

1. Hu B, Fan Z, Wei H, Song Y, Qiu R, Chen M, Xu W, Xue J, Wang F. Detection of mucoid enteropathy syndrome disease in rabbit farms in East China. Res Vet Sci. 2018; 119:259-261.

2. Marlier D, Dewrée R, Lassence C, Licois D, Mainil J, Coudert P, Meulemans L, Ducatelle R, Vindevogel H. Infectious agents associated with epizootic rabbit enteropathy: isolation and attempts to reproduce the syndrome. Vet J. 2006; 172(3):493-500.

3. Dong YF. Pathogens of Diarrhea in rabbits and its control measures. Chinese Journal of Rabbit Farming. 1998; 2: 3-5. (in chinese)

4. Petric M, Middleton PJ, Grant C, Tam JS, Hewitt CM. Lapine rotavirus: preliminary studies on epizoology and transmission. Can J Comp Med. 1978; 42(1): 143-147.

5. Ciarlet M, Gilger MA, Barone C, McArthur M, Estes MK, Conner ME. Rotavirus disease, but not infection and development of intestinal histopathological lesions, is age restricted in rabbits. Virology. 1998; 251(2): 343-360.

6. Peeters JE, Pohl P, Okerman L, Devriese LA. Pathogenic properties of Escherichia coli strains isolated from diarrheic commercial rabbits. J Clin Microbiol. 1984; 20(1): 34-39. 
7. Peeters JE, Geeroms R, Orskov F. Biotype, serotype, and pathogenicity of attaching and effacing enteropathogenic Escherichia coli strains isolated from diarrheic commercial rabbits . Infect Immun. 1988; 56(6): 1442-1448.

8. Borrelli L, Fioretti A, Ruggiero V, Santaniello A, Cringoli G, Ricci A, Barco L, Menna LF, Dipineto L. Salmonella typhimurium DT104 in farmed rabbits. J Vet Med Sci. 2011; 73(3): 385-387.

9. Djukovic A, Garcia-Garcera M, Martínez-Paredes E, Isaac S, Artacho A, Martínez J, Ubeda C. Gut colonization by a novel Clostridium species is associated with the onset of epizootic rabbit enteropathy. Vet Res. 2018; 49(1): 123.

10. Massacci FR, Magistrali CF, Cucco L, Curcio L, Bano L, Mangili P, Scoccia E, Bisgaard M, Aalbæk B, Christensen H. Characterization of Pasteurella multocida involved in rabbit infections. Vet Microbiol. 2018; 213: 66-72.

11. Pakandl M. Coccidia of rabbit: a review. Folia Parasitol. (Praha). 2009; 56(3): 153-66.

12. Jing F, Yin G, Liu X, Suo X, Qin Y. Large-scale survey of the prevalence of Eimeria infections in domestic rabbits in China . Parasitol. Res. 2012; 110 (4): 1495-1500.

13. Abdel-Gaber R, Ataya F, Fouad D, Daoud M, Alzuhairy S. Prevalence, Morphological and Molecular Phylogenetic Analyses of the Rabbit Pinworm, Passalurus ambiguus Rudolphi 1819, in the Domestic Rabbits Oryctolagus cuniculus. Acta Parasitol. 2019; 64(2): 316-330.

14. Zhang W, Shen Y, Wang R, Liu A, Ling H, Li Y, Cao J, Zhang X, Shu J, Zhang L.

Cryptosporidium cuniculus and Giardia duodenalis in rabbits: genetic diversity and possible zoonotic transmission. PLoS One. 2012; 7(2): e31262.

15. Zhang X, Qi M, Jing B, Yu F, Wu Y, Chang Y, Zhao A, Wei Z, Dong H, Zhang L.

Molecular Characterization of Cryptosporidium spp., Giardia duodenalis, and Enterocytozoon bieneusi in Rabbits in Xinjiang, China. J Eukaryot Microbiol. 2018; 65(6): 854-859.

16. Burgisser H. Is Saccharomycopsis guttulatus really pathogenic for the rabbit?. Pathol Microbiol. 1961; 24, 357-362. (in French)

17. Zierdt CH, Detlefson C, Muller J, Waggie KS. Cyniclomyces guttulatus (Saccharomycopsis guttulata)-culture, ultrastructure and physiology. Antonie Van Leeuwenhoek. 1988; 54, 357-366.

18. Hersey-Benner C. Diarrhea in a rabbit. Cyniclomyces guttulatus yeast. Lab Anim (NY). 2008; 37(8): 347-349.

19. Peters S, Houwers DJ. A cat with diarrhoea associated with the massive presence of Cyniclomyces guttulatus in the feces. Tijdschr. Diergeneeskd. 2009; 134(5): 198-199. (in Dutch)

20. Flausino G, Leal PD, McIntosh D, Amaral LG, Teixeira Filho WL, Flausino W, Lopes CW. Isolation and characterization of Cyniclomyces guttulatus (Robin) Van Der Walt and Scott, 1971 in dogs in Brazil. Curr Microbiol. 2012; 65(5): 542-546. 21. Mandigers PJ, Duijvestijn MB, Ankringa N, Maes S, van Essen E, Schoormans 
AH, German AJ, Houwers DJ. The clinical significance of Cyniclomyces guttulatus in dogs with chronic diarrhoea, a survey and a prospective treatment study. Vet Microbiol. 2014; 172(1-2): 241-247.

22. Winston JA, Piperisova I, Neel J, Gookin JL. Cyniclomyces guttulatus Infection in Dogs: 19 Cases (2006-2013). J Am Anim Hosp Assoc. 2016; 52(1): 42-51.

23. Kurtzman CP, Robnett CJ. Identification and phylogeny of ascomycetous yeasts from analysis of nuclear large subunit(26S) ribosomal DNA partial sequences. Antonie Van Leeuwenhoek. 1998; 73(4): 331-371.

24. Close B, Banister K, Baumans V, Bernoth EM, Bromage N, Bunyan J. Erhardt W, Flecknell P, Gregory N, Hackbarth H, Morton D, Warwick C. Recommendations for euthanasia of experimental animals: part 2. DGXT of the European Commission. Lab Anim. 1997; 31(1): 1-32.

25. Kyria BM, Martin WM. Cyniclomyces van der Walt \& D.B. Scott (1971) [A]// Cletus P.K., Jack W.F., Teun B.. The yeasts: a taxonomic study (fifth edition) . Elsevier BV. 2011; 357-359

26. Richle R, Scholer HJ. Saccharomycopsis guttulata in rabbits: cultural properties and possible significance. Pathol. Microbiol (Basel). 1961; 24:783-793. (in German).

27. Shi T, Bao G, Fu Y, Suo X, Hao L. A low-virulence Eimeria intestinalis isolate from rabbit (Oryctolagus cuniculus) in China: molecular identification, pathogenicity, and immunogenicity. Parasitol Res. 2014; 113(3): 1085-1090.

28. Shi T, Tao G, Bao G, Suo J, Hao L, Fu Y, Suo X. Stable Transfection of Eimeria intestinalis and Investigation of Its Life Cycle, Reproduction and Immunogenicity. Front Microbiol. 2016; 7: 807.

29. Han YX, Jiang X, Ge XG, An XK, Zhu ZD, Zhan RL, Ma JF, Li Y, Wang C, Xu LL. The epidemiological investigation and pathogenicity study on Cyniclomyces guttulatus of police dogs in Tianjin area. Journal of Tianjin Agricultural University. 2018; 25(4): 44-47. (in Chinese) 


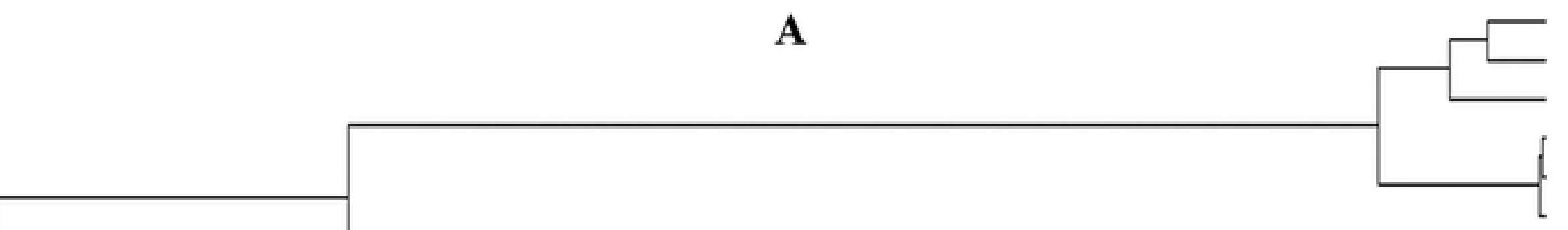
C. guttulatus NRRL Y-17561 C. guttulatus Dog-1 C. guttulatus DPA-CGR1 C. guttulatus CGDPA-GP1 C. guttulatus China isolate Nakaseomyces delphensis Saccharomyces cerevisiae 14.1

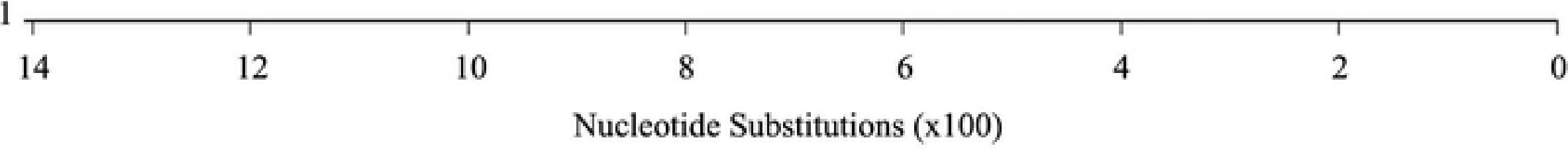
Pichia pastoris

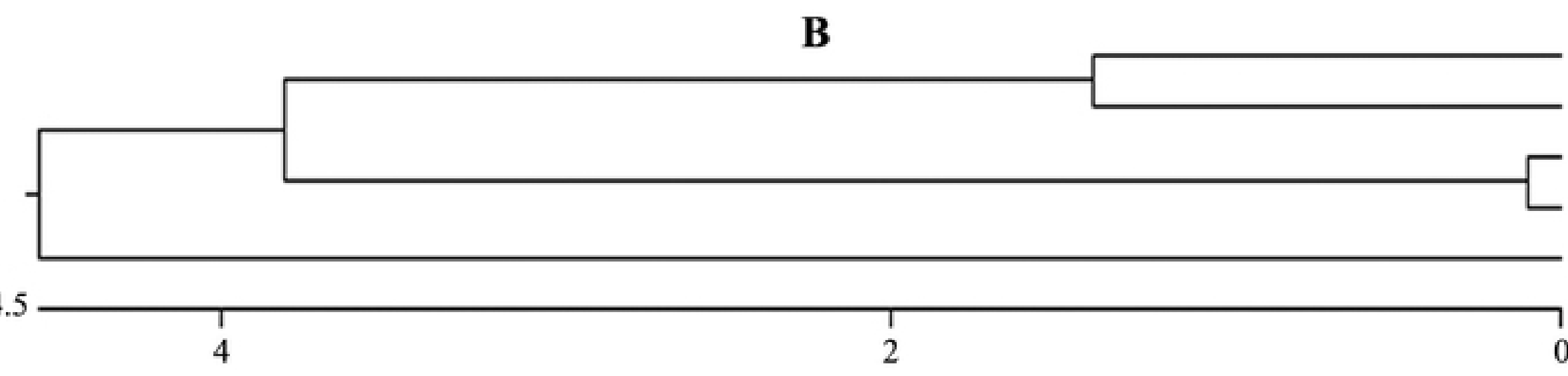

Nakaseomyces delphensis Saccharomyces cerevisiae C. guttulatus China isolate C. guttulatus NRRL Y-17561 Pichia pastoris

Nucleotide Substitutions (x100) Figure2 


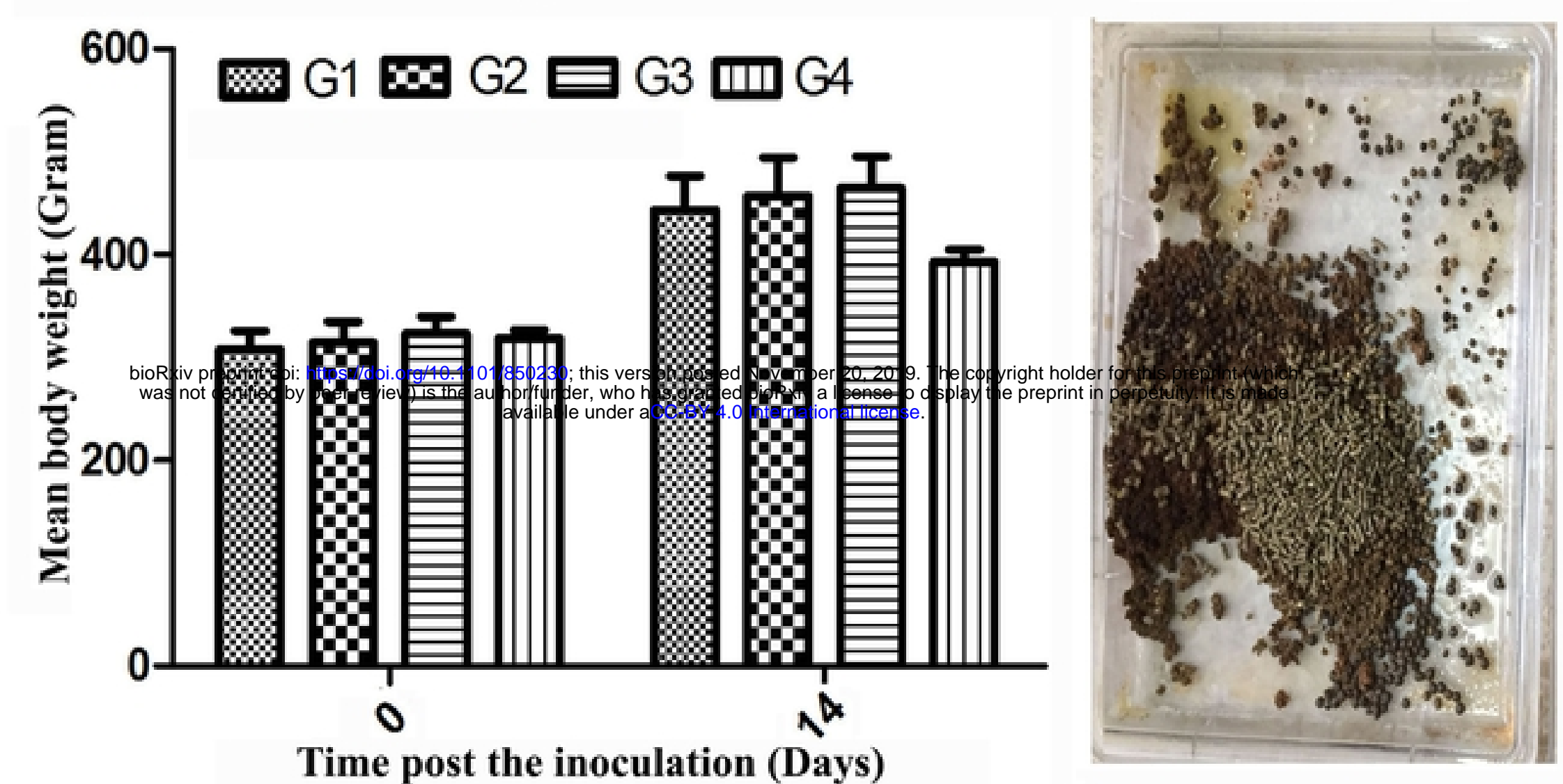

D

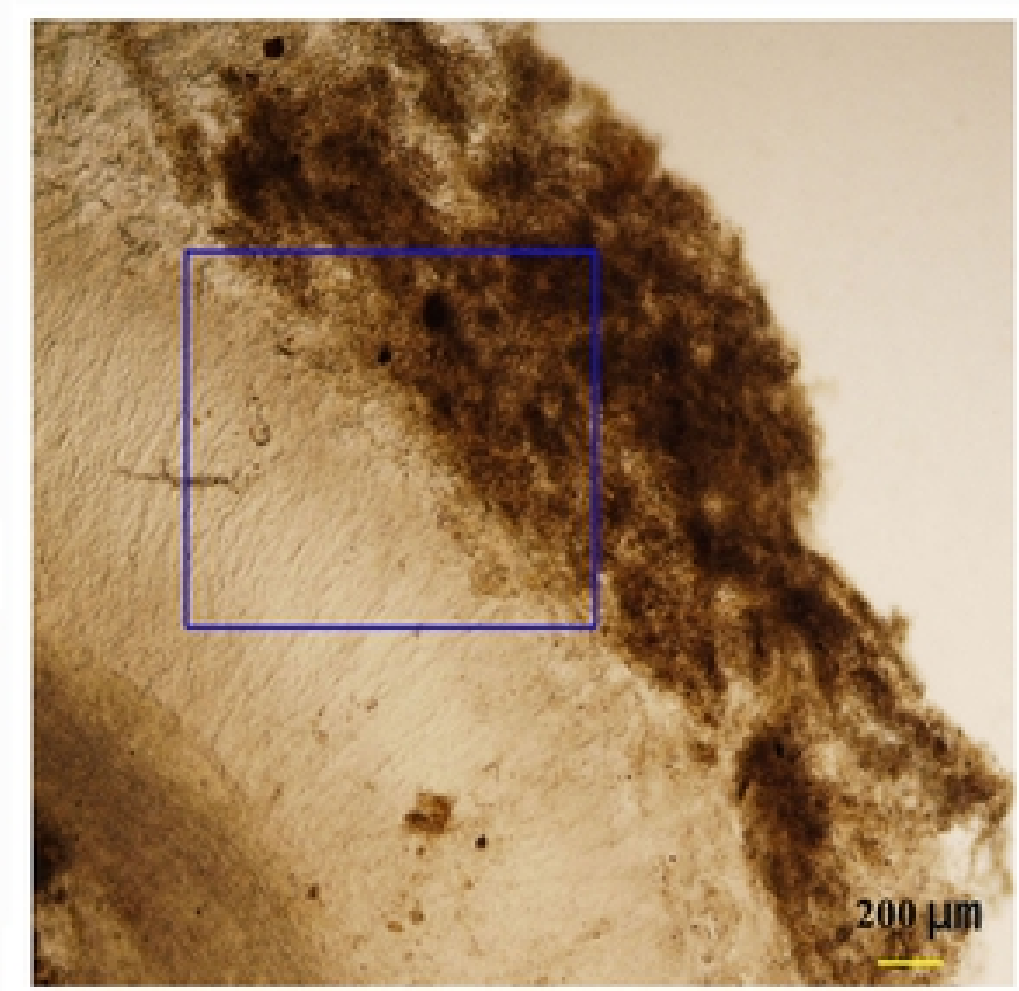

G

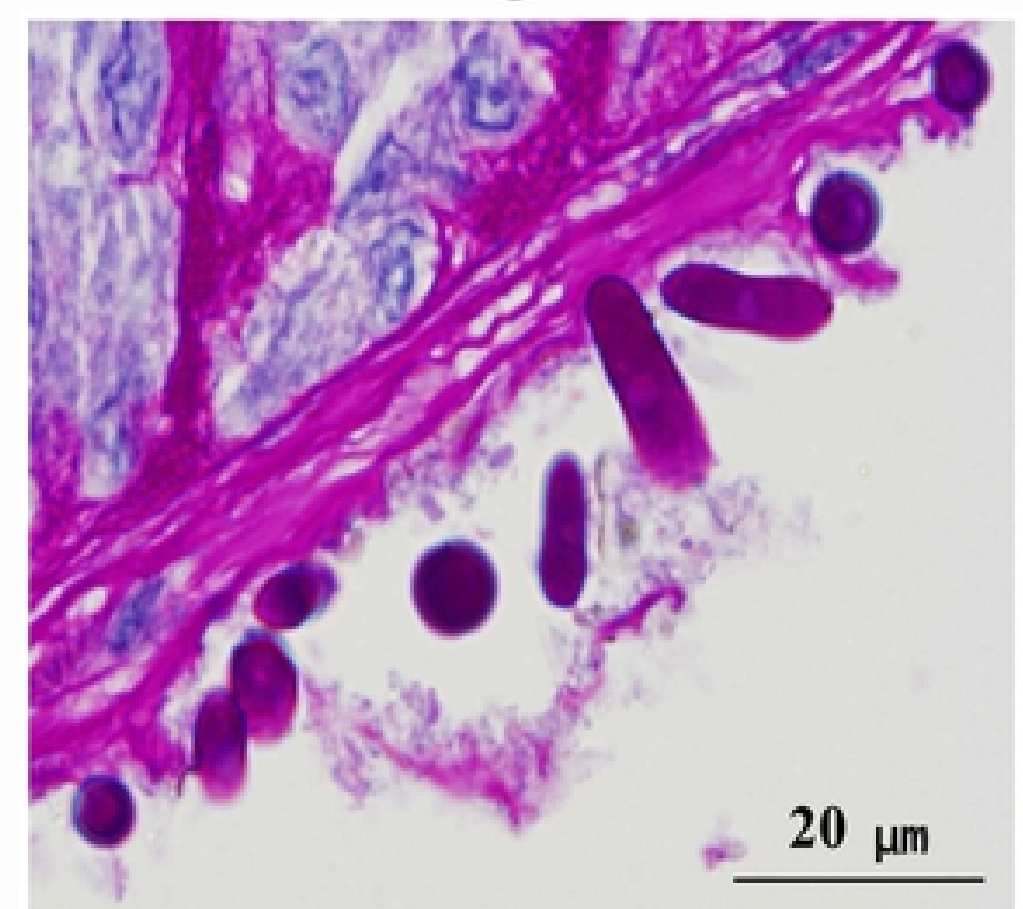

E

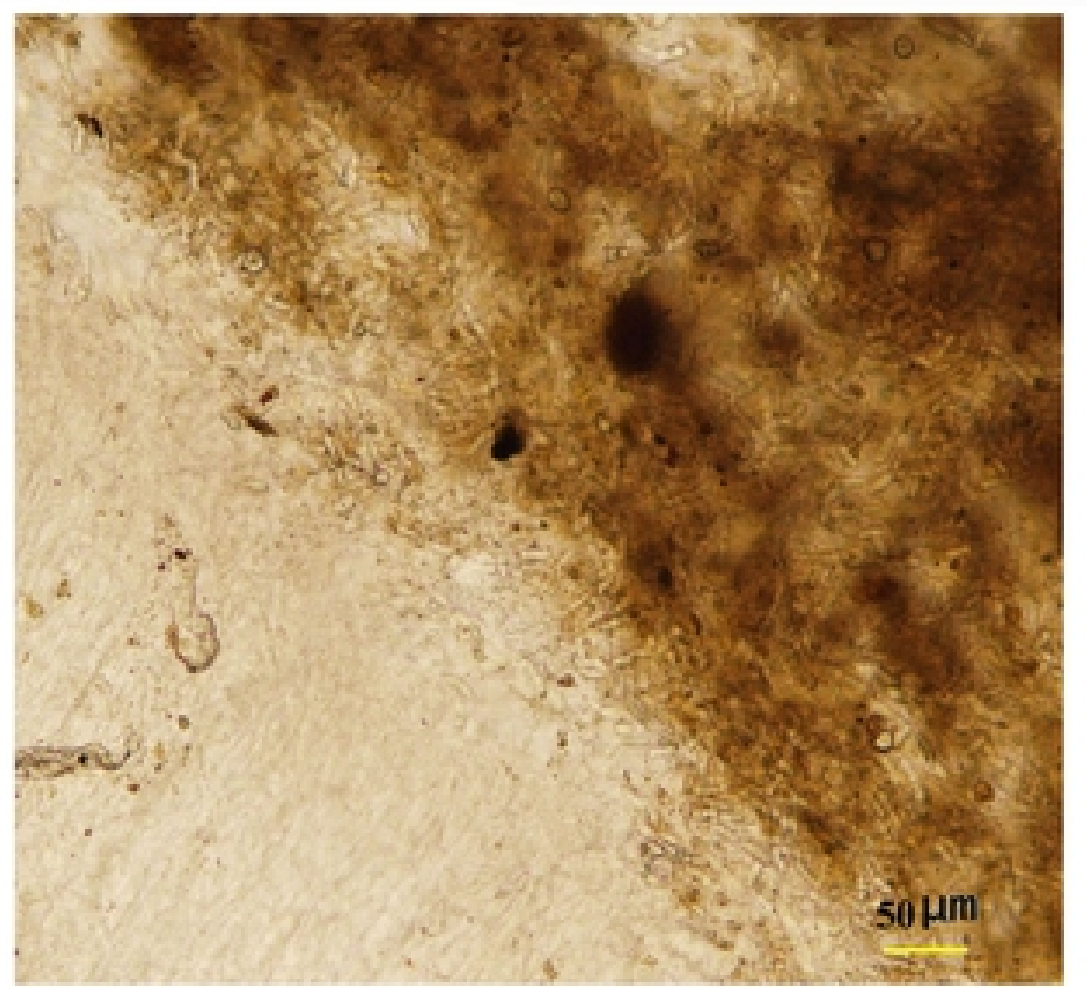

H

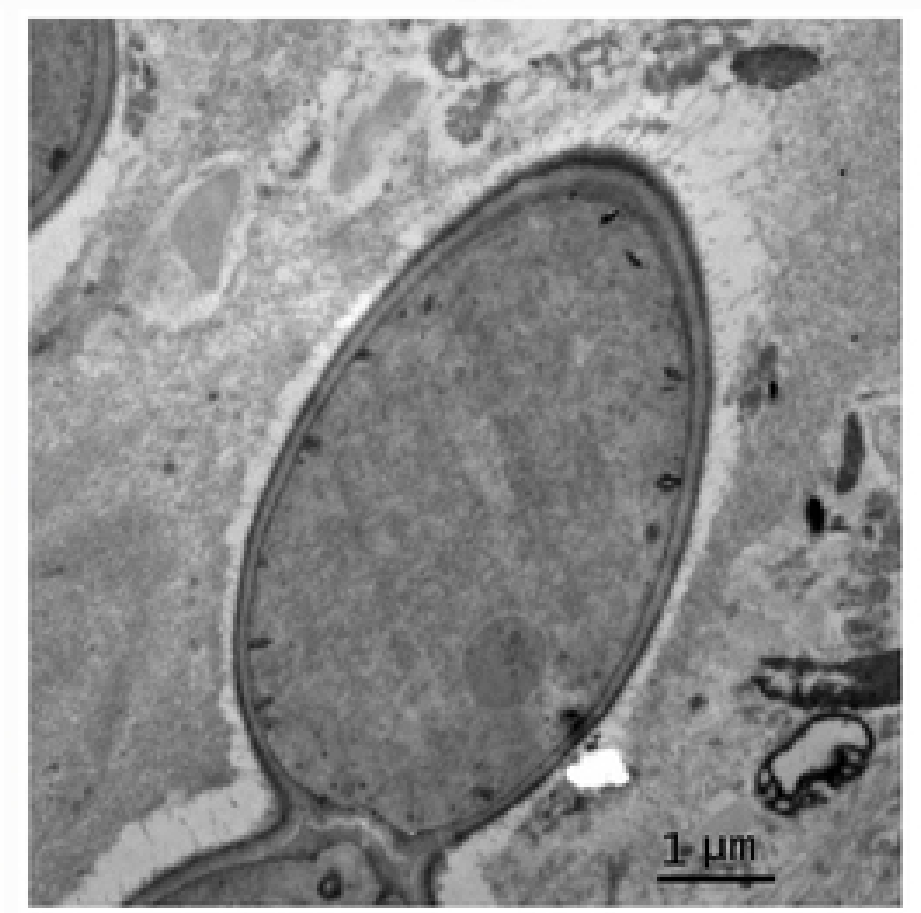

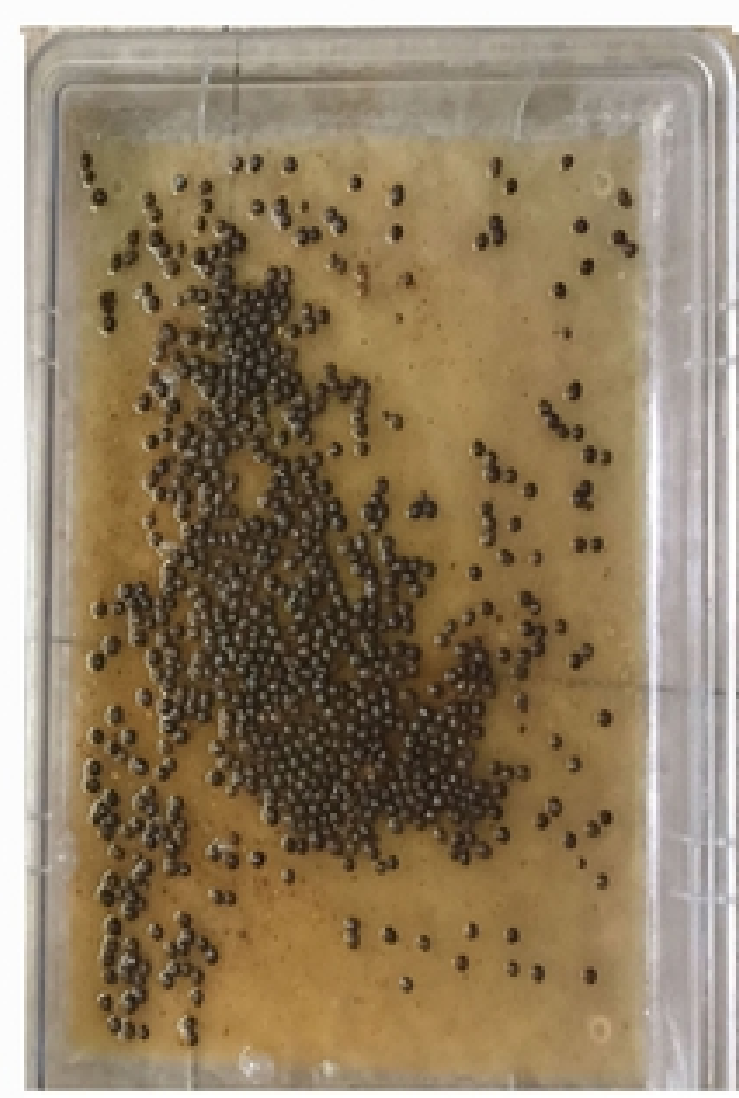

F

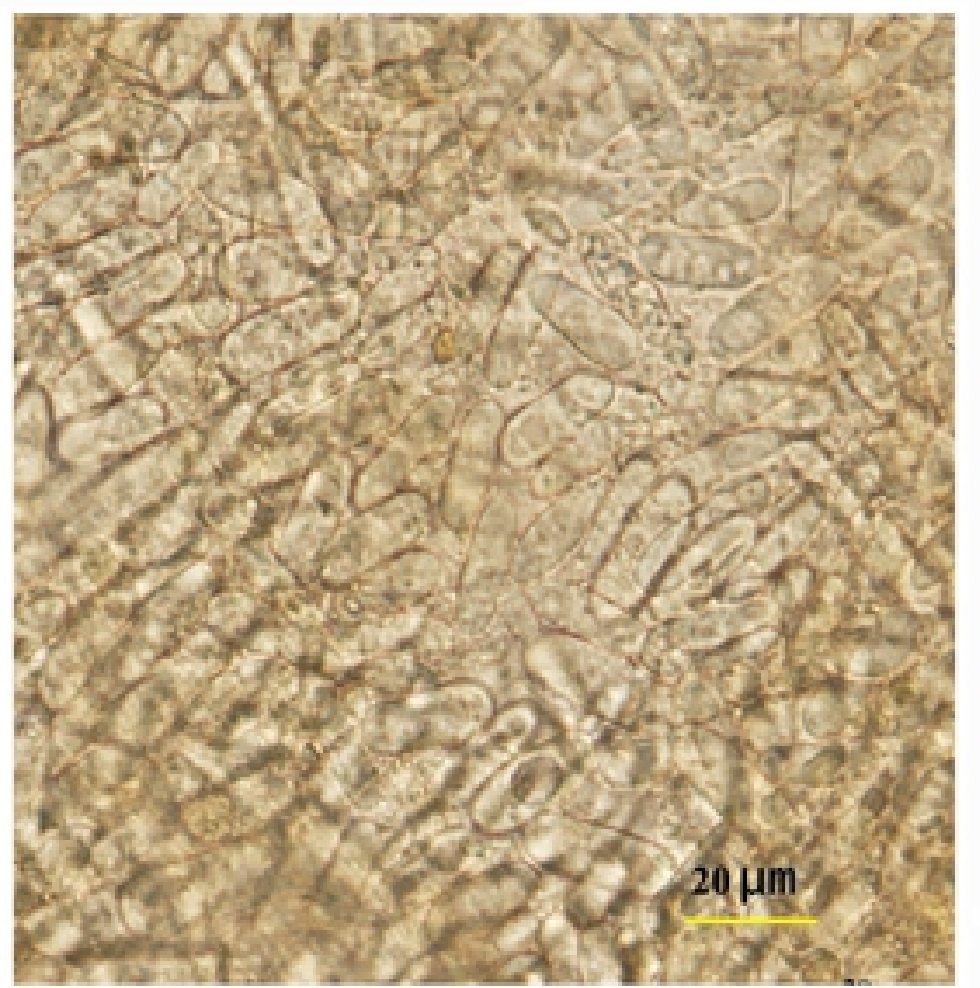

I

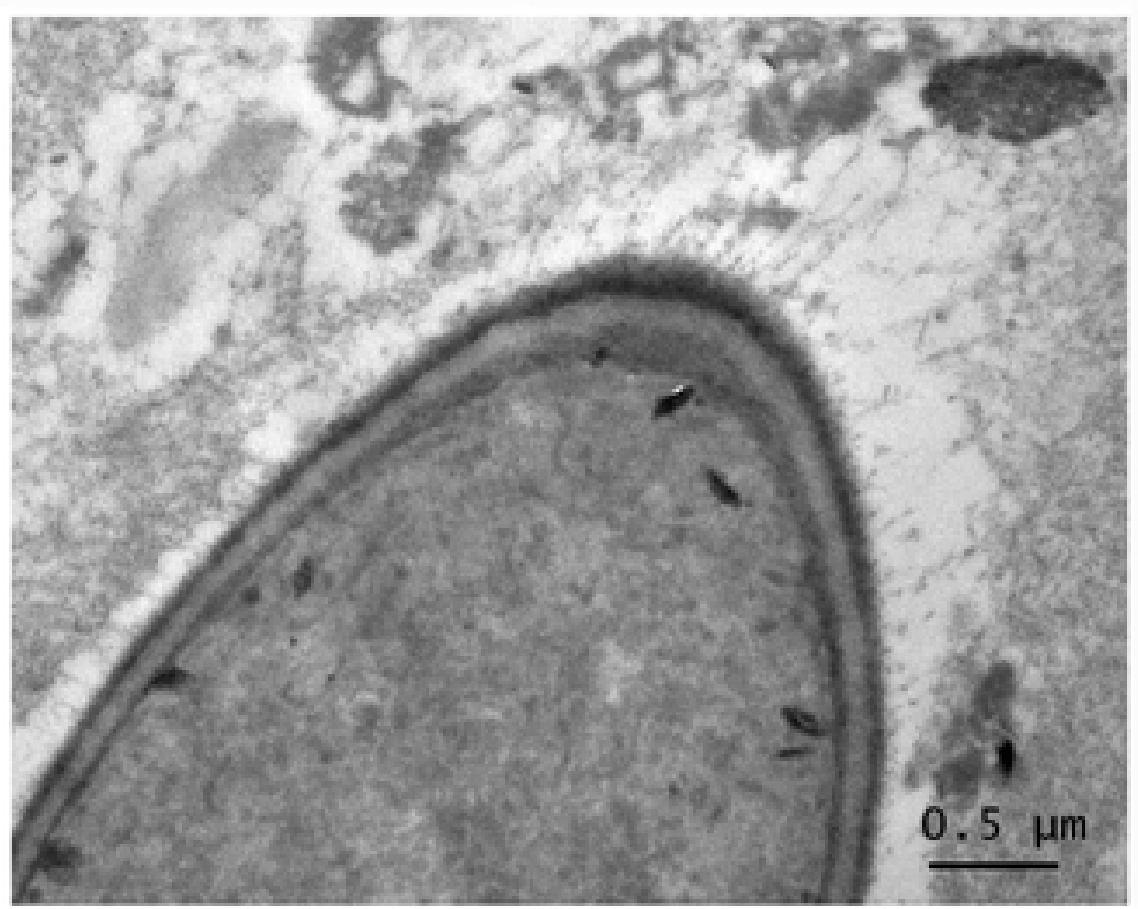

Figure3 
